\title{
Boolean Algebras and Distributive Lattices Treated Constructively ${ }^{1}$
}

\author{
John L. Bell
}

\begin{abstract}
Some aspects of the theory of Boolean algebras and distributive lattices -- in particular, the Stone Representation Theorems and the properties of filters and ideals -- are analyzed in a constructive setting.

KEYWORDS. Boolean algebra, distributive lattice, filter, constructive.

AMS CLASSIFICATION. 03B20, 06D99, 06E99

My purpose in this paper is to analyze some aspects of the theory of Boolean algebras and distributive lattices within a constructive context, in particular, without employing the law of excluded middle. Throughout, we work within a constructive set theory which, provided with a suitable type-theoretic formulation, can be interpreted within an arbitrary topos (see,e.g. [3]).
\end{abstract}

\section{PRELIMINARIES}

We employ the standard notation and terminology for Boolean algebras. If $\left(B, \bigvee_{B}, \bigwedge_{B}, \leq_{B}, 0_{B}, 1_{B}\right)$ is a Boolean algebra (we shall usually omit the subscript " $B$ "), we write $a \Rightarrow b$ for $a^{*} \vee b$ and $a \Leftrightarrow b$ for $(a \Rightarrow b) \wedge(b \Rightarrow a)$. Clearly $a \Leftrightarrow b=1$ iff $a=b$. We write 2 for the initial (two element) Boolean algebra $\{0,1\}$ and 1 for the trivial (one element) Boolean algebra: this is, up to isomorphism, the unique Boolean algebra $B$ in which $0_{B}=1_{B}$. We denote by BOOL the category of Boolean algebras and Boolean homomorphisms.

By a distributive lattice we shall understand such a lattice $\left(L, \bigvee_{L}, \bigwedge_{L}, \leq_{L}, 0_{B}, 1_{B}\right)$ (again, we shall usually omit the subscript " $L$ ") with top and bottom elements $0_{L}, 1_{L}$. Homomorphisms between distributive lattices in this sense will always be presumed to preserve 0 and 1: BOOL is a full subcategory of the category of distributive lattices and homomorphisms in this sense. A distributive lattice $L$ is a Heyting algebra if for each pair $a, b$ of elements of $L$ there is an element of $L$, which we denote by $a \Rightarrow b$, such that, for all $x \in L, x \wedge a \leq b$ iff $x \leq a \Rightarrow b$. (In a Boolean algebra the two definitions of $\Rightarrow$ are equivalent.) We write $a^{*}$ for $a \Rightarrow 0$. It is easily shown that a Heyting algebra is a Boolean algebra iff it satisfies either of the equivalent identities $x \vee x^{*}=1$, $x^{* *} \Rightarrow x=1$. A Heyting algebra is a Stone algebra if it satisfies the identity $x^{*} \vee x^{* *}=1$, or either of the equivalent identities $(x \wedge y)^{*}=x^{*} \vee y^{*},(x \vee y)^{* *}=x^{* *} \vee y^{* *}$.

\footnotetext{
${ }^{1}$ Mathematical Logic Quarterly 45, 1999.
} 
In constructive set theory the power set $\mathbf{P} X$ of any set $X$ is a Heyting algebra under the usual set-theoretic operations: $\cup$ (union), $\cap$ (intersection) and $\mathscr{C}$ (complement). In particular, writing 1 for the one-element set $\{0\}, \mathbf{P} 1$ is a Heyting algebra which we shall denote by $\Omega$. Each proposition $\alpha$ of constructive set theory is naturally correlated with the element $\widehat{\alpha}=\{x \in 1: \alpha\}$ of $\Omega$, and each element $\omega$ of $\Omega$ with the proposition $1 \in \omega$. The correspondence $\alpha \mapsto \hat{\alpha}$ has the property that $\hat{\alpha}=\hat{\beta}$ iff $\alpha$ and $\beta$ are equivalent. We shall follow the usual practice and identify $\widehat{\alpha}$ with $\alpha$; in that case the top element 1 of $\Omega$ is identified with the identically true proposition true and the bottom element $\varnothing$ of $\Omega$ with the identically false proposition false. These identifications explain why it is customary to call $\Omega$ the algebra of propositions. The following conditions are then equivalent: (i) $\Omega$ is a Stone algebra; (ii) for any proposition $\alpha, \neg \alpha$ or $\neg \neg \alpha$; (iii) $D e$ Morgan's law: for any propositions $\alpha, \beta, \neg(\alpha \& \beta) \rightarrow \neg \alpha$ or $\neg \beta$; (iv) for any propositions $\alpha, \beta$, $\neg \neg(\alpha$ or $\beta) \rightarrow \neg \neg \alpha$ or $\neg \neg \beta$. Also the following are equivalent: (i) $\Omega$ is a Boolean algebra; (ii) the law of excluded middle: for any proposition $\alpha, \alpha$ or $\neg \alpha$; (iii) the law of double negation: for any proposition $\alpha, \neg \neg \alpha \rightarrow \alpha$.

A subset $Y$ of a set $X$ is called stable if $\mathscr{C} \mathscr{C} Y=Y$, that is, if, for any $x \in X, \neg \neg(x \in Y) \rightarrow$ $x \in Y$; it is complemented if $Y \cup \mathscr{C} Y=X$, that is, if, for any $x \in X$, either $x \in Y$ or $\neg x \in Y$ : clearly any complemented set is stable (but not conversely). For any set $X$, the families $\mathbf{C} X$ and $\mathbf{S} X$ of complemented and stable subsets, respectively, of $X$ form Boolean algebras: the operations on the former are the usual set-theoretical ones; the same is true for the latter with the exception of $\vee$, which is defined to be the double complement of the union. We write $\Omega_{\neg \neg}$ for $\mathbf{S} 1$; and clearly $\mathbf{C} 1$ is (isomorphic to) the initial Boolean algebra 2.

A filter (resp., ideal) in a distributive lattice $L$ is a subset $F$ (resp., $I$ ) such that $1 \in F, x, y$ $\in F \rightarrow x \wedge y \in F, x \in F \& x \leq y \rightarrow y \in F($ resp. $0 \in I, x, y \in I \rightarrow x \vee y \in I, x \in I \& y \leq x \rightarrow$ $y \in I$.) A filter $F$ (ideal $I$ ) is proper if $0 \notin F(1 \notin I)$; clearly a distributive lattice is trivial iff it contains no proper filters (or no proper ideals). A filter $F$ (ideal $I$ ) in $L$ is prime if it is proper and satisfies the condition $x \vee y \in F \rightarrow x \in F$ or $y \in F(x \wedge y \in I \rightarrow x \in I$ or $y \in I)$ : if $L$ is a Boolean algebra, this is equivalent to the condition that, for any $x, x \in F$ or $x^{*} \in F\left(x \in I\right.$ or $\left.x^{*} \in I\right)$. Note that it follows immediately from this that both prime filters and prime ideals in Boolean algebras are complemented. It follows in turn that for each Boolean algebra $B$, there is a natural correspondence between prime filters (or ideals) and homomorphisms $B \rightarrow 2$ : each prime filter $P$ in $B$ is correlated with the homomorphism $h: B \rightarrow \mathbf{2}$ defined by $h(x)=1$ iff $x \in P$, and each homomorphism $h: B \rightarrow \mathbf{2}$ with the prime filter $h^{-1}[1]$. A filter (ideal) is an ultrafilter (maximal ideal) if it is proper and maximal with respect to that property. It is readily shown that a proper filter $F$ is an ultrafilter (maximal ideal) iff it satisfies the condition $\forall x[\forall y \in F(x \wedge y \neq 0 \rightarrow x \in F]$, and that a proper ideal $I$ is maximal iff it satisfies the condition $\forall x[\forall y \in I(x \vee y \neq 1 \rightarrow x \in I]$, In a 
Heyting algebra these conditions are easily shown to be equivalent to $\forall x\left[x \notin F \rightarrow x^{*} \in F\right]$ and $\forall x\left[x \notin I \rightarrow x^{*} \in I\right]$. We note that ultrafilters (and maximal ideals) in distributive lattices are stable. For it is readily shown that the double complement of a proper filter is a proper filter; thus, if $U$ is an ultrafilter, $\mathscr{C} \mathscr{C} U$ is a proper filter containing, and so identical with, $U$.

We shall employ the two following results, which are to be found in [5] and [6] respectively:

Result I. The following conditions are constructively equivalent ${ }^{2}$ :

(i) every ultrafilter in a distributive lattice is prime;

(ii) every ultrafilter in a Boolean algebra is prime;

(iii) $\Omega$ is a Stone algebra.

Result II. It is constructively provable that every distributive lattice can be embedded in a Boolean algebra.

\section{PROPERTIES OF FILTERS.}

In a constructive context the primeness property of filters "refracts" into a number of different properties, which we define below.

A filter $F$ in a distributive lattice $L$ is said to be:

almost prime if it is proper and, for all $x, y \in L, x \vee y \in F \& x \notin F \rightarrow y \in F$;

pseudoprime if $\mathscr{C} \mathscr{C} F$ is prime;

quasiprime if $\mathscr{C F}$ is a proper ideal;

coideal if $F=\mathscr{C I}$ for some proper ideal $I$;

comaximal if $F=\mathscr{C} M$ for some maximal ideal $M$.

Let $P, Q$ be properties of filters. We write $\mathbf{C}(P, Q)$ (resp. $\mathbf{C} *(P, Q))$ for the assertion "every filter in a distributive lattice (resp. Boolean algebra) possessing property $\mathrm{P}$ also possesses property Q." We also write

$P \rightarrow_{1} Q$ (resp. $\left.P \Rightarrow_{1} Q\right)$ for the assertion "C(P,Q) (resp. $\left.\mathbf{C}^{*}(P, Q)\right)$ is constructively provable;"

$P \rightarrow_{2} Q$ (resp. $\left.P \Rightarrow_{2} Q\right)$ for the assertion "C $(P, Q)$ (resp. $\left.\mathbf{C}^{*}(P, Q)\right)$ is constructively equivalent to the assertion that $\Omega$ is a Stone algebra;"

$P \rightarrow_{3} Q$ for the assertion $\mathrm{C}(P, Q)$ is equivalent to the assertion that $\Omega$ is a Boolean algebra."

${ }^{2}$ Actually, the result of [5] is stated for ideals rather than filters, but the two formulations are easily seen to be equivalent. 
2.1. Theorem. The various filter properties are related as follows: (i) prime $\Rightarrow_{1}$ ultra, (ii) prime $\Rightarrow_{1}$ pseudoprime, (iii) prime $\Rightarrow_{1}$ comaximal, (iv) prime $\rightarrow_{1}$ almost prime, (v) coideal $\Rightarrow_{1}$ comaximal, (vi) almost prime $\rightarrow_{1}$ quasiprime, (vii) almost prime $\Rightarrow_{1}$ ultra, (viii) ultra $\rightarrow_{1}$ almost prime, (ix) ultra $\rightarrow_{1}$ coideal, (x) coideal $\rightarrow_{1}$ almost prime, (xi) ultra $\rightarrow_{2}$ prime, (xii) ultra $\Rightarrow_{2}$ prime, (xiii) prime $\rightarrow_{2}$ pseudoprime, (xiv) ultra $\rightarrow_{2}$ pseudoprime, (xv) ultra $\Rightarrow 2$ pseudoprime, (xvi) quasiprime $\rightarrow_{2}$ pseudoprime, (xvii) quasiprime $\Rightarrow_{2}$ pseudoprime, (xviii) comaximal $\rightarrow_{2}$ prime, (xix) comaximal $\Rightarrow_{2}$ prime, $(\mathrm{xx})$ coideal $\rightarrow_{2}$ prime, (xxi) coideal $\Rightarrow_{2}$ prime, (xxii) almost prime $\Rightarrow_{2}$ prime, (xxiii) almost prime $\Rightarrow_{2}$ pseudoprime, (xxiv) prime $\rightarrow_{3}$ coideal, (xxv) quasiprime $\rightarrow_{3}$ coideal.

Proof. We write $L$ for a distributive lattice, $B$ for a Boolean algebra, $F$ for a proper filter, and $I$ for a proper ideal, in either.

(i). Let $F$ be prime in $B$. Then $\forall x\left[x \in F\right.$ or $\left.x^{*} \in F\right]$, whence $\forall x\left[x \notin F \rightarrow x^{*} \in F\right]$, so that $F$ is an ultrafilter.

(ii). If $F$ is prime in $B$, then $F$ is complemented, hence identical with its double complement, which is accordingly prime, so that $F$ is pseudoprime.

(iii). Let $F$ be prime in $B$. Then $\mathscr{C F}$ is an ideal, and indeed a prime ideal, since from $x \in F$ or $x^{*} \in F$ we get $x^{*} \notin F$ or $x \notin F$. A similar argument as in the proof of (i) shows that $\mathscr{C} F$ is maximal. Since $\mathrm{F}$ is complemented, $\mathscr{C} \mathscr{C} F=F$ and so $F$ is comaximal.

(iv) is obvious.

(v). If $F=\mathscr{C} I$ in $B$, then $x \notin I \rightarrow x \in F \rightarrow x^{*} \notin F \rightarrow x \in I$. So $I$ is maximal, and $F$ comaximal.

(vi). If $F$ is almost prime in $L$, then $x \vee y \in F \rightarrow(y \notin F \rightarrow x \in F)$, so $(x \notin F \& y \notin F) \rightarrow$ $\neg(y \notin F \rightarrow x \in F) \rightarrow x \vee y \notin F$. Therefore $\mathscr{C} F$ is an ideal.

(vii). If $F$ is almost prime in $B$, then since $x \vee x^{*}=1 \in F$, it follows that $x \notin F \rightarrow$ $x^{*} \in F$, so that $F$ is an ultrafilter.

(viii). Let $U$ be an ultrafilter in $L$, and suppose that $a \vee b \in U, a \notin U$. Consider 
$\{x \in L: a \vee x \in U\}$. This is a proper filter containing $U \cup\{b\}$, which, since $U$ is an ultrafilter, must coincide with $U$, so that $b \in U$. Therefore $a \vee b \in U \& a \notin U \rightarrow b \in U$, and $U$ is almost prime.

(ix). If $U$ is an ultrafilter in $L$, then, as we have seen, $U$ is almost prime, so that $a \notin U \&$ $b \notin U \rightarrow a \vee b \notin U$, and it follows that $\mathscr{C} U$ is a (proper) ideal. Since $U$ is stable, $U=\mathscr{C} \mathscr{C} U$, so that $U$ is coideal.

(x). Suppose $F=\mathscr{C} I$ in $L$. Then from $x \in I \& y \in I \rightarrow x \vee y \in I$ we get $x \vee y \notin I \rightarrow$ $\neg(x \in I \& y \in I)$. So

$$
\begin{aligned}
x \vee y \notin I \& \neg x \notin I & \rightarrow \neg(x \in I \& y \in I) \& \neg x \in I \\
& \rightarrow \neg[\neg(x \in I \& y \in I) \rightarrow x \notin I] \\
& \rightarrow \neg[x \in I \rightarrow(x \in I \& y \in I)] \\
& \rightarrow \neg[x \in I \rightarrow y \in I] \\
& \rightarrow \neg y \in I .
\end{aligned}
$$

Therefore $x \vee y \in F \& x \notin F \rightarrow y \in F$ and $F$ is almost prime.

(xi) and (xii) together constitute Result I.

(xiii). Suppose that $\Omega$ is a Stone algebra and that $F$ is prime in $L$. Then $\neg \neg(x \vee y \in F) \rightarrow$ $\neg \neg(x \in F$ or $y \in F) \rightarrow \neg \neg(x \in F)$ or $\neg \neg(y \in F)$. Therefore $F$ is pseudoprime.

Conversely, assume $\mathbf{C}$ (prime, pseudoprime). Then since $\{$ true $\}$ is obviously a prime filter in $\Omega$, it must be pseudoprime, that is, $\mathscr{C} \mathscr{C}\{$ true $\}$ is prime. It is easy to see that this is precisely the assertion that, for any proposition $\alpha$,

$$
\neg \neg(\alpha \vee \beta) \rightarrow \neg \neg \alpha \vee \neg \neg \beta,
$$

which is equivalent to the condition that $\Omega$ be a Stone algebra.

(xiv) and (xv). If $\Omega$ is a Stone algebra, then by the two immediately preceding results, $\mathbf{C}($ ultra, prime) and $\mathbf{C}$ (prime, pseudoprime), whence $\mathbf{C}($ ultra, pseudoprime).

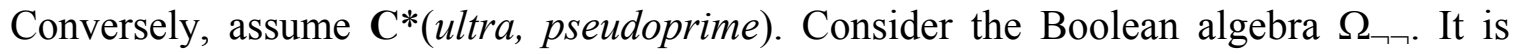
easy to see that $\{$ true $\}$ is the sole proper filter therein, and so is an ultrafilter. Then $\mathscr{C} \mathscr{C}\{$ true $\}=$ $\left\{\right.$ true\} is prime, that is, for $\alpha, \beta$ in $\Omega_{\neg \neg}$,

$$
\alpha \vee_{\neg \neg} \beta \rightarrow \alpha \text { or } \beta,
$$

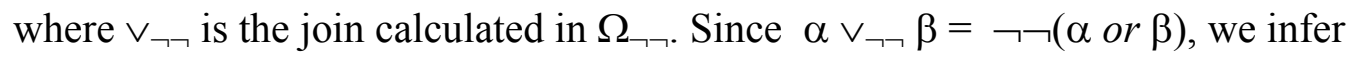

$$
\neg \neg(\alpha \text { or } \beta) \rightarrow \alpha \text { or } \beta,
$$

Now for arbitrary $\alpha, \beta$ in $\Omega, \neg \neg \alpha, \neg \neg \beta$ are in $\Omega_{\neg \neg}$, so it follows that 


$$
\neg \neg(\alpha \text { or } \beta) \rightarrow \neg \neg(\neg \neg \alpha \text { or } \neg \neg \beta) \rightarrow \neg \neg \alpha \text { or } \neg \neg \beta,
$$

and therefore $\Omega$ is a Stone algebra.

(xvi). First assume that $\Omega$ is a Stone algebra, and that $\mathscr{C} F$ is an ideal in $L$. Then $x \notin F \&$ $y \notin F \rightarrow x \vee y \notin F$, whence

$$
\neg \neg(x \vee y \in F) \rightarrow \neg(x \notin F \& y \notin F) \rightarrow \neg \neg(x \in F) \text { or } \neg \neg(y \in F) .
$$

Thus $\mathscr{C} \mathscr{C} F$ is pseudoprime.

Conversely, suppose that $\mathbf{C}$ (quasiprime, pseudoprime). Then, since (by (viii) and (vi)) ultra $\rightarrow_{1}$ quasiprime, it follows that $\mathbf{C}($ ultra, pseudoprime). Since ultrafilters are stable, we conclude from this that $\mathbf{C}$ (ultra , prime), which by Result $\mathbf{I}$ implies that $\Omega$ is a Stone algebra.

(xvii). The argument here is similar to that for (xvi).

(xviii) - (xxi). If $\Omega$ is a Stone algebra, and $F=\mathscr{C} I$ in $L$, then $x \vee y \in F \rightarrow x \vee y \notin I \rightarrow$ $\neg(x \in I \& y \in I) \rightarrow \neg(x \in I)$ or $\neg(y \in I) \rightarrow x \in F$ or $y \in F$. Hence $F$ is prime. Conversely assume $\mathbf{C}^{*}$ (comaximal, prime). Consider the Boolean algebra $\Omega_{\neg}$. It is easy to see that $\{$ false is the sole proper ideal therein, and so, a fortiori, maximal. Therefore $\{$ true $\}=\mathscr{C}\{$ false $\}$ is prime in $\Omega_{\neg \rightarrow}$, and as in the proof of (xiv) we conclude that $\Omega$ is a Stone algebra.

(xxii) and (xxiii) . These follow from (xii), (xv) and the (above established) fact that in Boolean algebras almost prime filters coincide with ultrafilters.

(xxiv). Assume $\mathbf{C}$ (prime, coideal). In $\Omega$, \{true $\}$ is a prime filter, and so coideal. Since it is easily verified that $\{$ false $\}$ is the sole proper ideal in $\Omega$, it follows that $\{$ true $\}=\mathscr{C}\{$ false $\}$. But this means that, for any proposition $\alpha, \neg \neg \alpha \rightarrow \alpha$, so that $\Omega$ is a Boolean algebra.

(xxv). This follows from (xxiv) and (iv).

Note that, if $\Omega$ itself is a Boolean algebra, then all the conditions of Thm. 2.1 coincide for filters in arbitrary Boolean algebras.

A theorem of Nachbin (see, e.g. [1]) asserts that (assuming Zorn's lemma ${ }^{3}$ ), if every

\footnotetext{
${ }^{3}$ It should be noted that, as shown in [4], Zorn's lemma is "constructively neutral" in the sense that it has no purely logical consequences, that is, it has no effect on the properties of $\Omega$. It is therefore to be contrasted with its classical equivalent the axiom of choice which is well-known to imply that $\Omega$ is a Boolean algebra.
} 
prime filter in a distributive lattice $L$ is an ultrafilter, then $L$ is a Boolean algebra (and of course conversely). Actually the proof of the result does not really involve primeness per se, but rather the (classically) stronger property that we have termed comaximality. So, for the record, we state and prove in a classical setting

Nachbin's Theorem. If every comaximal filter in a distributive lattice $L$ is an ultrafilter, then $L$ is a Boolean algebra.

Proof. Let $a \in L$ and suppose that $a$ has no complement in $L$. Then, using Zorn's lemma, the set $\{x \in L: x \wedge a=0\} \cup\{a\}$ is included in some maximal ideal $M$. Then $F=\mathscr{C} M$ is comaximal, hence an ultrafilter, and $a \notin F$. But, for $x \in L, x \in F \rightarrow x \wedge a \neq 0$, so, since $F$ is an ultrafilter, $a \in F$. This contradiction shows that, classically, $a$ must have had a complement after all; since this is the case for arbitrary $a \in L$, the latter is a Boolean algebra.

If $L$ is a Heyting algebra in which every comaximal filter is an ultrafilter, the proof of Nachbin's theorem shows that $x \vee x^{*}=1$ for any $x \in L$. If the same argument is carried out constructively, however, we can only conclude that $\neg \neg\left(x \vee x^{*}=1\right)$ for any $x \in L$, so that $L$ is what we might call near Boolean. Note that it does not follow from this that $L$ is Boolean, because it is easy to see that $\Omega$ is always near Boolean. In fact, Nachbin's theorem (stated in the form above) itself implies that $\Omega$ is a Boolean algebra, since $\Omega$ satisfies the premise of that theorem. For if $F$ is comaximal in $\Omega$, then, in view of the fact that $\{\mathbf{f a l s e}\}$ is the only proper ideal in $\Omega, F=\mathscr{C}\{$ false $\}$ and the latter is easily shown to be (the only) ultrafilter in $\Omega$.

\section{THE STONE REPRESENTATION THEOREM.}

Recall that the classical Stone Representation Theorem for Boolean algebras asserts that every Boolean algebra is isomorphic to a subalgebra of $\mathbf{P} S$ for some set $S$. In a constructive context, we observe that since every member of a Boolean algebra of subsets of a set is obviously complemented, in the statement of this theorem "PS" may be replaced by "CS".

We call a distributive lattice (in particular, a Boolean algebra) semisimple if the intersection of the family of all its prime filters is $\{1\}$. A Boolean algebra $C$ is said to be a cogenerator in BOOL if it has the following property: for any pair of parallel morphisms $f, g$ : $A \rightarrow B$ in BOOL, if $h \circ f=h \circ g$ for all $h: B \rightarrow C$, then $f=g$.

3.1. Theorem. The following assertions are constructively equivalent.

(i) The Stone Representation Theorem for Boolean algebras;

(ii) the Stone Representation Theorem for distributive lattices: any distributive lattice is isomorphic to a lattice of subsets of a set; 
(iii) any distributive lattice is semisimple;

(iv) any Boolean algebra is semisimple;

(v) The initial Boolean algebra $\mathbf{2}$ is a cogenerator in BOOL.

Proof. (i) $\rightarrow$ (ii). One direction is obvious. Since, by Result II, any distributive lattice is constructively embeddable in a Boolean algebra, (i) $\rightarrow$ (ii) follows immediately.

(ii) $\rightarrow$ (iii). Assume (ii); then any distributive lattice $L$ may be considered a sublattice of PS for some set $S$. For any $x \in S, F_{x}=\{X \in L: x \in X\}$ is a prime filter; if $X \in \cap\left\{F_{x}: x \in S\right\}$, then $x \in X$ for all $x \in S$, whence $X=S$. Therefore $\bigcap\left\{F_{x}: x \in S\right\}=\{S\}$, and $L$ is semisimple.

Conversely, assume (iii). Given a distributive lattice $L$, let $S$ be the set of all prime filters in $L$, and define $h: L \rightarrow \mathbf{P} S$ by $h(x)=\{F \in S: x \in F\}$. It is easy to see that $h$ is a homomorphism; the semisimplicity of $L$ implies that $h$ is injective. Hence (ii).

(i) $\rightarrow$ (iv). The proof of this is similar to that of (ii) $\rightarrow$ (iii).

(iv) $\rightarrow(\mathrm{v})$. Assume (iv) and suppose that $f, g: A \rightarrow B$ are such that if $h \circ f=h \circ g$ for all $h: B \rightarrow \mathbf{2}$. Then for all $h: B \rightarrow \mathbf{2}$ and $x \in A$ we have $h(f(x))=h(g(x))$ so that $1=h(f(x)) \Leftrightarrow h(g(x))$ $=h(f(x)) \Leftrightarrow h(g(x))$. Under the natural correspondence between homomorphisms $B \rightarrow \mathbf{2}$ and prime filters in $B$, this means that $f(x) \Leftrightarrow g(x)$ is contained in every prime filter in $B$. Since $B$ is semisimple, it follows that $f(x) \Leftrightarrow g(x)=1$, so that $f(x)=g(x)$ for every $x \in A$, i.e. $f=g$. Hence (v).

Conversely, assume (v). Consider the 4-element Boolean algebra

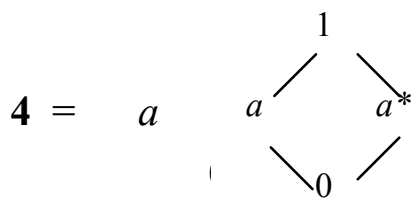

For any Boolean algebra $B$, each homomorphism $4 \rightarrow B$ is uniquely determined by the image of $a$, which can be an arbitrary element $b$ of $B$. Denote this homomorphism by $b^{\sim}$. Suppose now that every prime filter in $B$ contains $b$. Then, under the natural correspondence between prime filters in $B$ and homomorphisms $B \rightarrow \mathbf{2}$, this means that $h(b)=h(1)$, whence $h \circ b^{\sim}=h \circ 1^{\sim}$ for all $h: B \rightarrow \mathbf{2}$. By (v), $b^{\sim}=1^{\sim}$, so that $b=1$, and $B$ is semisimple.

In [2], it is shown that the Stone Representation Theorem for Boolean algebras condition (i) of 3.1 -implies, within any localic topos, that $\Omega$ is a Boolean algebra. We 
strengthen this result by showing that this condition - and hence also any one of the equivalent conditions of 3.1-implies, within the general context of constructive set theory, that $\Omega$ is a Boolean algebra.

3.2. Theorem. Any of (i) - (v) of Thm. 3.1 constructively implies that $\Omega$ is a Boolean algebra.

Proof. Let us assume, for instance, (iv). For each Boolean algebra $B$, let $\operatorname{Prim}(B)$ be the set of prime filters in $B$. Then $\bigcap \operatorname{Prim}(B)=\{1\}$ and we have

$$
\text { (*) } \quad \operatorname{Prim}(B)=\varnothing \rightarrow B \text { is trivial. }
$$

For if $B$ is trivial, it has no proper filters, so that $\operatorname{Prim}(B)=\varnothing$. Conversely, if $\operatorname{Prim}(B)=\varnothing$, then $\{1\}=\bigcap \operatorname{Prim}(B)=\bigcap \varnothing=B$, so that $B$ is trivial.

Now let $\alpha$ be any proposition, and define

$$
B_{\alpha}=\{\omega \in \Omega: \omega=\alpha \text { or } \omega=\text { true }\} .
$$

This is easily shown to be a Boolean algebra in which $0=\alpha, 1=$ true, meets are conjunctions, joins are disjunctions, and the complement of $\omega$ is $(\omega \rightarrow \alpha)$. Clearly

$$
(* *) \quad B_{\alpha} \text { is trivial } \rightarrow \alpha \text {. }
$$

Putting $(*)$ and $(* *)$ together, we see that

$$
\alpha \rightarrow \operatorname{Prim}\left(B_{\alpha}\right)=\varnothing \rightarrow \neg \exists X . X \in \operatorname{Prim}\left(B_{\alpha}\right) .
$$

Thus $\alpha$ is equivalent to a negated statement, so that $\neg \neg \alpha \rightarrow \alpha$. Since $\alpha$ was arbitrary, it follows that $\Omega$ is a Boolean algebra.

Thm. 3.1 can also be stated and proved, in a similar way, for nontrivial Boolean algebras and distributive lattices. However, the proof that any one of the correspondingly weakened versions of conditions (i) - (v) implies that $\Omega$ is a Boolean algebra differs from the proof of Thm. 3.2, as witness:

3.3. Theorem. The assertion any nontrivial Boolean algebra is semisimple constructively implies that $\Omega$ is a Boolean algebra.

Proof. Let $B$ be a semisimple Boolean algebra. Then $\{1\}$, as the intersection of prime filters, is the intersection of complemented sets and is therefore (as is easily seen), stable. So the premise of the present Theorem implies that $\{1\}$ is a stable subset of every nontrivial Boolean algebra. Now, by Result II, $\Omega$ is embeddable in a — necessarily nontrivial — Boolean algebra $B$, so we may consider $\Omega$ as a subset of $B$. Then $\{1\}=\{$ true $\}$ is a stable subset of $B$ and hence also of $\Omega$. But the stability of $\{$ true $\}$ in $\Omega$ is obviously equivalent to the assertion that it be a Boolean 
algebra.

Classically, the Stone Representation Theorem is equivalent to the assertion that $\mathbf{2}$ be injective ${ }^{4}$ in BOOL. As noted in [2], this equivalence is not constructively valid, since while the former can hold only when $\Omega$ is a Boolean algebra, the latter can be true even when $\Omega$ is merely a Stone algebra. (To see that the injectivity of $\mathbf{2}$ implies that $\Omega$ is a Stone algebra, observe that from this assumption it follows that the Boolean algebra $\Omega_{\neg}$ must have a homomorphism to 2 , and hence must also contain a prime filter. Since $\{$ true $\}$ is the only proper filter in $\Omega_{\neg,}$, it must be prime, and we have already observed (in the proof of Thm. 2.1) that this condition implies that $\Omega$ is a Stone algebra.)

In conclusion, we show that the injectivity of $\mathbf{2}$ is constructively equivalent to a number of familiar results in the theory of Boolean algebras. If $X$ is a set, we write $X \not \equiv \varnothing$ for $\exists x . x \in X$.

3.4. Theorem. The following are constructively equivalent (and each implies that $\Omega$ is a Stone algebra).

(i) For any Boolean algebra $B$ and any $x \neq 0$ in $B$ there is $h: B \rightarrow \mathbf{2}$ such that $h(x)=1$.

(ii) For any Boolean algebra $B$ and any $x \neq 0$ in $B$ there is a prime filter in $B$ containing $x$.

(iii) Any nontrivial Boolean algebra contains a prime filter.

(iv) Each proper filter in a Boolean algebra is contained in a prime filter.

(v) 2 is injective in BOOL.

(vi) For any Boolean algebra $B$, there is a set $S$ and a homomorphism $h: B \rightarrow \mathbf{P} S$ such that, for any $x \in B, x \neq 0 \rightarrow h(x) \not \equiv \varnothing$.

Proof. (i) $\rightarrow$ (ii) $\rightarrow$ (iii) are all obvious.

(iii) $\rightarrow$ (iv). Assume (iii) and let $F$ be a proper filter in a Boolean algebra $B$. Then the quotient $B / F$ is nontrivial and so contains a prime filter $P$. The inverse image $\pi^{-1}[P]$ of $P$ under the canonical homomorphism $\pi: B \rightarrow B / F$ is easily seen to be a prime filter in $B$ containing $F$.

(iv) $\rightarrow$ (v). Assume (iv), let $A$ a subalgebra of a Boolean algebra $B$, and let $h$ be a homomorphism of $A$ to 2 . Then $h^{-1}[1]$ is a (prime) filter in $A$ in turn generating a proper filter in $B$ which, by (iv), is contained in a prime filter $P$ in $B$. The homomorphism $B \rightarrow \mathbf{2}$ naturally corresponding to $P$ is an extension of $h$.

\footnotetext{
${ }^{4}$ A Boolean algebra $C$ is injective (in BOOL) if any homomorphism to $C$ from a subalgebra of any Boolean algebra $B$ can be extended to the whole of $B$.
} 
(v) $\rightarrow$ (iii). Assume (v) and let $B$ be a nontrivial Boolean algebra. Then 2 may be considered a subalgebra of $b$ and the identity homomorphism $\mathbf{2} \rightarrow \mathbf{2}$ has an extension to $B$, giving rise to a naturally correlated prime filter in $B$.

(iv) $\rightarrow$ (vi). Assume (iv), and let $S$ be the set of prime filters in a given Boolean algebra $B$. Define $h: B \rightarrow \mathbf{P} S$ by $h(x)=\{F \in S: x \in F\}$. This $h$ is a homomorphism; if $x \neq 0$ in $B$, then $x$ generates a proper filter which is contained in a prime filter $P$. Then $P \in h(x)$ and $h(x) \neq \equiv$. Hence (vi).

(vi) $\rightarrow$ (ii). Assume (vi) and the data of (ii). If $a \neq 0$ in $B$, then $h(a) \neq \equiv \varnothing$, so there is an element $s \in h(a)$. Then $\{x \in B: s \in h(x)\}$ is a prime filter in $B$ containing $a$. (ii) follows.

\section{REFERENCES}

[1] Balbes, R., and Dwinger, P., Distributive Lattices. University of Missouri Press, 1974.

[2] Banaschewski, B., and Bhutani, K., Boolean algebras in a localic topos. Math. Proc. Camb. Phil. Soc. 100 (1986), 43-55.

[3] Bell, J.L., Toposes and Local Set Theories: An Introduction. Clarendon Press, Oxford, 1988.

[4] Bell, J.L., Zorn's lemma and complete Boolean algebras in intuitionistic type theories. Forthcoming, Journal of Symbolic Logic.

[5] Johnstone, P.T., Another condition equivalent to De Morgan's law. Comm. Alg. 7 (12) (1979), 1309-1312.

[6] Peremans, W., Embedding of a distributive lattice into a Boolean algebra. Indag. Math. 19 (1957), 73-81. 